\title{
Benefícios do cateter central de inserção periférica em pacientes onco- lógicos na pediatria: revisão integrativa
}

\author{
Benefits of central peripheral insertion catheter in oncology patients in pediatrics: integra- \\ tive review
}

Beneficios del catéter central de inserción periférica en pacientes oncológicos en la pediatría: revisión integrativa

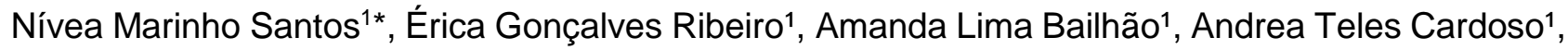
Aglaya Almeida Oliveira Lima Cordeiro ${ }^{1}$.

\section{RESUMO}

Objetivo: Revisar na literatura científica os benefícios do cateter central de inserção periférica no tratamento em crianças com câncer. Métodos: Trata-se de uma revisão literária integrativa, realizada por meio das bases de dados, Literatura Latino-Americana e do Caribe em Ciências da Saúde (LILACS), Medical Literature Analysis and Retrieval System Online (MedLine) e Scientific Electronic Library Online (SciELO), sendo utilizado artigos dos últimos 5 anos. Resultados: Nos 12 artigos selecionados, observou-se como benefícios do cateter central de inserção periférica na oncologia pediátrica, a possiblidade de infusão da nutrição parenteral total prolongada e medicamentos vesicantes e irritantes (83,3\%), via para terapias prolongadas $(66,6 \%)$, garantia de acesso vascular confiável (58,3\%), administração de hemoderivados e/ou transfusão de sangue, erradicação de complicações potenciais, inserção à beira leito, melhoria dos estágios dolorosos $(41,6 \%)$, facilidade da punção, menor custo e menor risco de contaminação $(33,4 \%)$, continuidade do tratamento a nível ambulatorial( $25 \%$ ), baixa trombogenicidade e conservação do sistema vascular periférico das extremidades $(16,6 \%)$, inserção com anestesia local e uso de múltiplos medicamentos $(8,3 \%)$. Conclusão: Com isso se faz necessário o treinamento e capacitação dos profissionais devidamente habilitados para passagem do dispositivo tendo em vista a diminuição das incidências no quadro de Infecções relacionadas à Assistência à Saúde (IRAS).

Palavras-chave: Cateterismo Periférico, Oncologia, Criança Hospitalizada.

\begin{abstract}
Objective: Review in scientific literature the benefits of the central peripheral insertion catheter in the treatment of cancer patients. Methods: It is an integrative literary review, carried out through the data bases, Latin American and Caribbean Literature in Health Sciences (LILACS), Medical Literature Analysis and Retrieval System Online (MedLine) and Scientific Electronic Library Online (SciELO), articles of the last 5 years have been used. Results: The 12 selected articles, observed as benefits of the central catheter of peripheral therapy in pediatric oncology, a possibility of prolonged total parenteral treatment and vesicant and irritant medications (83.3\%), for prolonged therapies (66.6\%). ), confined vascular access (58.3\%), administration of blood products and / or blood transfusion, correction of complications, border bed filling, improvement of pain stages $(41.6 \%)$, ease of puncture $(33.4 \%)$, maintenance of the outpatient treatment system $(25 \%)$, low thrombogenicity and preservation of the peripheral vascular system of the extremities (16.6\%), follow-up with local anesthesia and use of physicians $(8.3 \%)$. Conclusion: This will require the training and qualification of professionals who are properly qualified to pass the device in order to reduce the incidence of Infections related to Health Care (IRAS).
\end{abstract}

Key words: Peripheral Catheterization, Oncology, Hospitalized child.

\footnotetext{
${ }^{1}$ Centro Universitário Jorge Amado (UNIJORGE), Salvador-Bahia. * E-mail: nivea.marinhosantos@hotmail.com
} 


\section{RESUMEN}

Objetivo: Revisar en la literatura científica del catéter central de inserción periférica en el tratamiento a pacientes con cáncer. Métodos: Se trata de una revisión literaria integrativa, realizada a través de las bases de datos, Literatura Latinoamericana y del Caribe en Ciencias de la Salud (LILACS), Medical Literature Analysis and Retrieval System Online (MedLine) y Scientific Electronic Library Online (SciELO), se utilizaron artículos de los últimos 5 años. Resultados: Los 12 artículos seleccionados, observados como beneficios del catéter central de terapia periférica en la oncología pediátrica, una posibilidad de tratamiento parenteral total prolongado y medicamentos vesicantes e irritantes (83,3\%), vía para terapias prolongadas $(66,6 \%)$.), garantía de acceso vascular confinado (58,3\%), administración de hemoderivados y / o transfusión de sangre, corrección de complicaciones, llenado del lecho de borde, mejora de las etapas dolorosas $(41,6 \%)$, facilidad de la punción menor riesgo (33,4\%), mantenimiento del sistema de tratamiento ambulatorio $(25 \%)$, baja trombogenicidad y conservación del sistema vascular periférico de las extremidades (16,6\%), acompañamiento con anestesia local y uso de médicos $(8,3 \%)$. Conclusión: Con ello se hace necesario el entrenamiento y capacitación de los profesionales debidamente habilitados para pasar del dispositivo teniendo en vista la disminución de las incidencias en el cuadro de infecciones relacionadas à la Asistencia de la salud (IRAS).

Palabras clave: Cateterismo Periférico, Oncología, Niños Hospitalizados.

\section{INTRODUÇÃO}

O primeiro registro de câncer se deu no início do séc. VII a.C. no Egito, em que um sunu (médico da época) descreveu uma "massa proeminente no peito". No entanto até o séc. XIX havia poucos informes referentes à doença em decorrência de mortes precoces com enfermidades da época. Com a chegada da civilização moderna, no meado do séc. XX o câncer tornou-se evidente em razão do aumento da taxa de sobrevida humana, com isso foi intensificado estudos pertinentes à patologia (KARAM, 2015).

Segundo o Instituto Nacional do Câncer José Alencar Gomes da Silva (INCA), o câncer infanto-juvenil corresponde a um grupo de patologias que tem em comum a multiplicação de células anômalas que podem ocorrer em qualquer local do corpo humano. As neoplasmas mais frequentes na infância e adolescência são as leucemias (doença que afeta os glóbulos brancos), os do Sistema nervoso central (SNC) e linfomas (Hodgkin e não- Hodgkin) (BRASIL, 2017).

Estima-se que entre os anos de 2018-2019 no Brasil, ocorrerão 12.500 casos novos de câncer em crianças e adolescentes de 0 a 19 anos, com maior incidência na região Sudeste e Nordeste com números de 6.050 e 2.750 respectivamente (BRASIL, 2018).

A assistência oncológica desenvolve-se por intermédio dos cuidados preventivo, curativo e paliativo. Porém, neste estudo, destaca-se o cuidado curativo, pelo qual é constituído por diagnóstico e tratamento da doença (SANTOS et al., 2013). Onde os tratamentos mais utilizados são a quimioterapia, radioterapia, cirurgia e o transplante de medula óssea (RIBEIRO et al., 2016).

A terapia endovenosa é indispensável para assistência à saúde, devido aos pacientes que fazem tratamento por períodos prolongados como administração de medicamentos vesicantes, irritantes e antibióticos, provocarem danos sérios de visualização do vaso e acesso venoso. Na oncologia o cateter central de inserção periférica (PICC), vem sendo utilizado com maior frequência tendo em vista a menor incidência de riscos e complicações (VERA et al., 2015).

O uso do PICC foi retratado pela primeira vez em 1929, pelo médico alemão Werner Theodor Otto Forssman, ao introduzir uma Cânula em sua própria veia antecubital. No território Brasileiro seu uso iniciou-se no ano de 1990 na área de neonatologia e propagou-se para as áreas de terapia intensiva, oncologia e cuidados domiciliares (SANTO et al., 2017).

Comparado a outros cateteres o PICC apresenta melhor relação de benefícios: baixo custo, pode ser inserido em beira leito ou a domicílio excluindo o uso de centro cirúrgico, exigindo apenas um Raio-X para confirmação de sua localização, contribuindo positivamente na qualidade de vida dos pacientes, pois resulta na redução da dor física e psicológica (GONÇALVES et al., 2017). 
Estima-se que no Brasil a incidência no quadro de infecção por via sanguínea através do cateter central de inserção periférica é em torno de 14\% a 16\% ocupando o terceiro lugar como motivo de infecção hospitalar (BRASIL, 2013). Esta baixa ocorrência está relacionada à via de passagem do cateter que se localiza na região dos membros superiores onde se há baixa concentração de agentes patogênicos, em comparação a outros cateteres que estão expostos a secreções endotraqueais e nasais (NEGELISKII et al., 2017).

O cateter intravenoso central periférico é constituído por materiais bioestáveis e biocompatíveis, a base de silicone e poliuretano de baixa capacidade trombogênica, introduzido através de um vaso periférico e instalado na veia cava superior ou inferior, sendo a veia basílica de primeira escolha, seguida da veia cefálica (OLIVEIRA et al., 2014).

A implantação do PICC é um processo de alta complexidade que exige capacitação específica. No Brasil a sua prática é realizada exclusivamente por enfermeiros e médicos, adequadamente capacitados por meio de um curso regulamentado. A resolução $n^{\circ} 258 / 2001$ habilita o profissional de enfermagem devidamente habilitado a realização da implantação do cateter central de inserção periférica (MOURA et al., 2013).

O uso do cateter central de inserção periférica em unidades de oncologia pediátrica promove uma melhor qualidade da assistência prestada ao cliente, tanto em ambiente intra-hospitalar como extra-hospitalar, proporcionando aos portadores do dispositivo a redução de agentes estressores que causam impactos dolorosos e psicológicos (MOURA et al., 2013). A questão norteadora sobre a temática escolhida foi: quais os benefícios do cateter central de inserção periférica na oncologia pediátrica? Diante do exposto, esse artigo tem como objetivo descrever os benefícios da utilização do cateter central de inserção periférica em pacientes oncológicos na pediatria de acordo com a revisão literária.

\section{METODOLOGIA}

Trata-se de uma revisão de literatura de caráter integrativo com o intuito de descrever a veracidade sem interferir em seus resultados permitindo agregação de dados da literatura teórica, sendo constituído pela procura, coleta e análise de informações a fim de solidificar os benefícios apresentados pelo PICC em pacientes oncológicos pediátricos.

A identificação de artigos inclui as bases de dados MEDLINE (Medical Literature Analysis and Retrieval System Online), LILACS (Literatura Latino-Americana e do Caribe em Ciências da Saúde) e SciELO (Scientific Electronic Library Online). Os descritores utilizados foram: "Cateterismo Periférico", "Oncologia" e "Criança Hospitalizada". Dos 20 artigos coletados no período de agosto a novembro, utilizou-se 12 que atendia aos seguintes critérios: estudos contemplados com os descritores acima referidos, disponíveis na íntegra de forma gratuita, publicados no período de 2013 a 2018, nos idiomas em português, inglês e espanhol. Foram excluídos 08 artigos de revisão e demais estudos que após a leitura de títulos, resumos e texto completo, se encontravam duplicados e não contemplaram o tema proposto.

Em relação aos aspectos éticos o presente estudo por se tratar de uma revisão de literatura não será enviado ao Comitê de Ética em Pesquisa de acordo com a Resolução 510/2016 do Conselho Nacional de Saúde (CNS), contudo todos os princípios éticos estabelecidos no que se refere a zelar pela legitimidade das informações, privacidade e sigilo das informações, quando necessárias, tornando os resultados desta pesquisa públicos.

\section{RESULTADOS E DISCUSSÃO}

As temáticas nos 12 periódicos sobre os benefícios do PICC na oncologia pediátrica selecionados, em 4 artigos (33,4\%) identificavam o contexto das publicações cientificas sobre o PICC, 3 (25\%) artigos abordam as indicações para uso do PICC, nos outros 3 artigos (25\%) relatam o benefício atribuído após implementação do uso do PICC e $2(16,6 \%)$ discorrem sobre as orientações na manutenção do PICC aos pacientes no ambiente extra-hospitalar (Quadro 1). 
Quadro 1: Periódicos selecionados para analise acerca do cateter central de inserção periférica em pacientes oncológicos pediátricos no período de 2013 a 2018.

\begin{tabular}{|c|c|}
\hline Artigo & Objetivo \\
\hline $\begin{array}{l}\text { A atuação do enfermeiro na } \\
\text { prática de inserção e manu- } \\
\text { tenção do PICC: uma revi- } \\
\text { são integrativa de literatura } \\
\text { (VERA et al., 2015) }\end{array}$ & $\begin{array}{l}\text { Analisar literaturas científi- } \\
\text { cas referentes a atuação do } \\
\text { profissional de enfermagem } \\
\text { na inserção e manutenção } \\
\text { do PICC. }\end{array}$ \\
\hline $\begin{array}{l}\text { Cateteres venosos centrais } \\
\text { de inserção periférica: alter- } \\
\text { nativa ou primeira escolha } \\
\text { em acesso vascular? } \\
\text { (SANTO et al., 2017) }\end{array}$ & $\begin{array}{l}\text { Evidenciar os efeitos da in- } \\
\text { serção de PICCs ecoguia- } \\
\text { dos e fixados por fluorosco- } \\
\text { pia realizado no Hospital e } \\
\text { Maternidade São Luiz } \\
\text { (HMSL). }\end{array}$ \\
\hline $\begin{array}{l}\text { Custo Benefício Do Cateter } \\
\text { Central De Inserção Perifé- } \\
\text { rica em comparação com o } \\
\text { Cateter Venoso Central } \\
\text { (NEGELISKIl et al., 2017) }\end{array}$ & $\begin{array}{l}\text { Demonstrar os benefícios } \\
\text { do PICC diante do seu } \\
\text { baixo custo e menores } \\
\text { complicações, comparados } \\
\text { ao cateter venoso central. }\end{array}$ \\
\hline
\end{tabular}

Resultados

Pôde se observar que $90,90 \%$ dos periódicos que discorrem sobre os cuidados na inserção e manutenção do PICC, são específicos da área de enfermagem, constando um elevado número de publicações comparados a outros profissionais como da área médica.

O estudo aponta que 256 acessos vasculares foram solicitados sendo 236 PICCs, com a veia basílica direita sendo a primeira escolha precedida pela braquial direita. As indicações foram devido ao uso de antibiótico prolongado, acesso venoso de difícil acesso e nutrição parenteral prolongada.

Pôde se observar menores gastos com recursos materiais e humanos na passagem do PICC, o dispositivo pode ser inserido pelo enfermeiro e não requer a presença do cliente no centro cirúrgico para inserção, diminuindo as taxas de infecção via cateter. Enquanto o cateter venoso central é um dispositivo que se faz necessário o uso do bloco cirúrgico sendo sua passagem exclusiva pelo médico elevando o custo para a inserção, e as taxas de infecção.

Cateter central de inserção periférica em pediatria e neonatologia: possibilidades de sistematização em hospital universitário (OLIVEIRA et al., 2014)

Cateter central de inserção periférica: revisão bibliométrica (MOURA et al., 2013)

Competência e Habilidade do Enfermeiros para realização do PICC em crianças (SANTOS et al., 2017)

"Peripherally Inserted Central Catheters: Our Experience from a Cancer Research Centre" (SUNDRIYAL et al., 2014)
Discorrer sobre estruturação do uso do PICC no cotidiano de um hospital escola.
O conhecimento teórico dos enfermeiros sobre o PICC é de extrema importância na avaliação da indicação, inserção, manutenção e remoção do mesmo. Além disso, é necessário a implementação da SAE para instruir os pacientes na preservação do acesso após a alta hospitalar utilizando o PICC, de formar que facilite a continuidade do atendimento em nível ambulatorial, evitando novas inserções do PICC.

Identificar a quantidade, 0 ano e o contexto de publicações cientificas no brasil em enfermagem, inerentes ao tema PICC.

Discorrer as ideias impostas nas publicações cientificas sobre a segurança, competência e a prática dos enfermeiros na inserção do PICC em crianças.

Orientar aos pacientes oncológicos o cuidado com o cateter de inserção periférica para diminuição das complicações.
Identificou que $93,10 \%$ dos estudos selecionados eram dissertações de mestrado, os anos e a região de maiores quantitativos de publicações deu-se entre 2004 a 2008 na região sudeste. Sobre as temáticas pertinentes abordadas foram o conhecimento teórico prático dos enfermeiro sobre o PICC e a infecção provocada pelo mesmo.

O enfermeiro é apto para a implantação do PICC, através de cursos capacitantes, porém a prática de inserção, manutenção e remoção está diretamente relacionado com o progresso benéfico do manejo do PICC.

A manutenção do PICC é explicada em uma ficha de cuidados fornecida ao paciente. Isso inclui informações sobre técnicas assépticas ao manipular o cateter, curativos semanais e lavagem do cateter quando não estão em uso e evitar manuseio desnecessário, tais condutas ajudam a evitar complicações relacionadas ao dispositivo. 


\begin{tabular}{|c|c|c|}
\hline \multicolumn{3}{|l|}{ Continuação Quadro 1.} \\
\hline Artigo & Objetivo & Resultados \\
\hline $\begin{array}{l}\text { Utilização do cateter central } \\
\text { de inserção periférica em } \\
\text { neonatologia (JANTSCH et } \\
\text { al., 2014) }\end{array}$ & $\begin{array}{l}\text { Definir a implantação do } \\
\text { PICC em unidades de neo- } \\
\text { natologia, evidenciando os } \\
\text { benefícios em RNs com } \\
\text { baixo peso e prematuros. }\end{array}$ & $\begin{array}{l}\text { Os autores constataram que os RNs com baixo } \\
\text { peso com percentual de } 37 \% \text { e prematuros } 69 \% \\
\text { obtiveram sucesso nas inserções com o PICC } \\
\text { desde a primeira tentativa, utilizando a veia cefá- } \\
\text { lica e a temporal. }\end{array}$ \\
\hline $\begin{array}{l}\text { Catéter central de acceso } \\
\text { periférico en paciente pedi- } \\
\text { átrico con cáncer: estudio } \\
\text { de caso (RANGEL e FAN- } \\
\text { DIÑO, 2015) }\end{array}$ & $\begin{array}{l}\text { Avaliar o uso do PICC no } \\
\text { paciente oncológico pediá- } \\
\text { trico e a repercussão da } \\
\text { adesão no bem-estar des- } \\
\text { ses pacientes. }\end{array}$ & $\begin{array}{l}\text { Subsequente ao estudo foi observado que o uso } \\
\text { do PICC gerou diminuição da dor e ansiedade, efe- } \\
\text { tividade na adesão e ausência de sinais de infec- } \\
\text { ção durante } 100 \text { dias de utilização. }\end{array}$ \\
\hline $\begin{array}{l}\text { Utilização do cateter central } \\
\text { de inserção periférica na } \\
\text { unidade de terapia intensiva } \\
\text { neonatal (LOPES et al., } \\
\text { 2015) }\end{array}$ & $\begin{array}{l}\text { Evidenciar com base em ar- } \\
\text { tigos as indicações clínicas } \\
\text { para a aplicação do PICC e } \\
\text { os benefícios da sua utiliza- } \\
\text { ção na UTIN. }\end{array}$ & $\begin{array}{l}\text { Nas indicações do uso PICC englobou recém-nas- } \\
\text { cidos de extremo baixo peso e prematuros que ne- } \\
\text { cessitam de tratamento prolongado na unidade de } \\
\text { terapia intensiva neonatal, tendo necessidades de } \\
\text { infusões de soluções para auxílio na sua terapêu- } \\
\text { tica. Diante deste fato a instituição que foi realizado } \\
\text { o estudo, evidenciou-se como vantagens menores } \\
\text { números de punções, diminuição nos atrasos das } \\
\text { medicações e maior relação custo-risco benefício. }\end{array}$ \\
\hline $\begin{array}{l}\text { Peripherally inserted cen- } \\
\text { tral catheters in non-hospi- } \\
\text { talized cancer patients: } 5- \\
\text { year results } \\
\text { of a prospective study (CO- } \\
\text { TOGNI et al., 2015) }\end{array}$ & $\begin{array}{l}\text { Mensurar a credibilidade do } \\
\text { uso do PICC em pacientes } \\
\text { oncológicos não hospitali- } \\
\text { zados que requerem de te- } \\
\text { rapias intravenosas prolon- } \\
\text { gadas. }\end{array}$ & $\begin{array}{l}\text { O estudo constata que o manuseio correto do } \\
\text { PICC confere segurança para os pacientes onco- } \\
\text { lógicos não hospitalizados que realizam tratamen- } \\
\text { tos prolongados. Logo foi registrado que as infec- } \\
\text { ções da corrente sanguínea tiveram incidência ate- } \\
\text { nuada, assim como nos casos de trombose e com- } \\
\text { plicações mecânicas. Salientado também a longa } \\
\text { taxa de permanecia do cateter e uma baixa possi- } \\
\text { bilidade da retirada devido a complicações. }\end{array}$ \\
\hline $\begin{array}{l}\text { Focus on peripherally in- } \\
\text { serted central catheters in } \\
\text { critically ill patients (CO- } \\
\text { TOGNI e PITTIRUTI, 2014) }\end{array}$ & $\begin{array}{l}\text { Relatar técnicas na inser- } \\
\text { ção e o cuidado dos profis- } \\
\text { sionais de enfermagem no } \\
\text { manuseio do PICC em pa- } \\
\text { cientes críticos }\end{array}$ & $\begin{array}{l}\text { Os profissionais de enfermagem devem obedecer } \\
\text { às recomendações constadas nas diretrizes que } \\
\text { expõem a inserção e os cuidados com o PICC. O } \\
\text { enfermeiro deve inserir o cateter em veias antecu- } \\
\text { bital ou cefálica localizadas na região do braço, es- } \\
\text { tando atentos para a não formação de trombose } \\
\text { venosa periférica. O estudo demonstra, que uma } \\
\text { das ações para se evitar infecções relacionadas ao } \\
\text { cateter é o cuidado asséptico durante a troca de } \\
\text { curativo, tal competência deve ser realizada exclu- } \\
\text { sivamente pela equipe de enfermagem devida- } \\
\text { mente habilitada. }\end{array}$ \\
\hline
\end{tabular}

Fonte: Dados obtidos através da análise dos artigos elegidos na revisão de literatura.

Na representação do Organograma 1 em conformidade com os artigos elegidos, os benefícios relacionados ao PICC na oncologia pediátrica foram observados desde a inserção até o tempo de permanência do cateter. Dentre eles podemos mencionar nutrição parenteral prolongada diminuindo o risco de contaminação e medicamentos vesicantes e irritantes repetindo-se em 10 artigos (83,3\%), terapêutica prolongada em 8 artigos (66,6\%), garantia de acesso vascular confiável em 7 artigos (58,3\%), administração de hemoderivados e/ou transfusão de sangue, elimina complicações potenciais, inserido à beira do leito, melhoria dos estágios dolorosos e desconforto em 5 artigos (41,6\%), facilidade da punção, menor custo e menor risco de contaminação em 4 artigos (33,4\%), continuidade do tratamento a nível ambulatorial em 3 artigos (25\%), baixa trombogenicidade e conservação do sistema vascular periférico das extremidades em 2 artigos (16,6\%), inserção com anestesia local e uso de múltiplos medicamentos em 1 artigo $8,3 \%$. 
Uma soma de benefícios foram percebidos na utilização do PICC a partir da década de 1970, quando iniciou a implementação do cateter para infundir nutrição parenteral em unidade de terapia intensiva neonatal e se fortaleceu em outros setores beneficiando pacientes desde neonatos a adultos decorrente da possibilidade do uso prolongado nas terapias de longa duração, administração de medicamentos vesicantes, irritantes ou vasoativos; soluções hiperosmolares ou com pH não fisiológico e administração de quimioterápicos, conforme a Infusion Nursing Society (OLIVEIRA et al., 2014).

Organograma 1: Apresenta o quantitativo dos benefícios do cateter central de inserção periférica abordados nos artigos selecionados $(n=12)$.

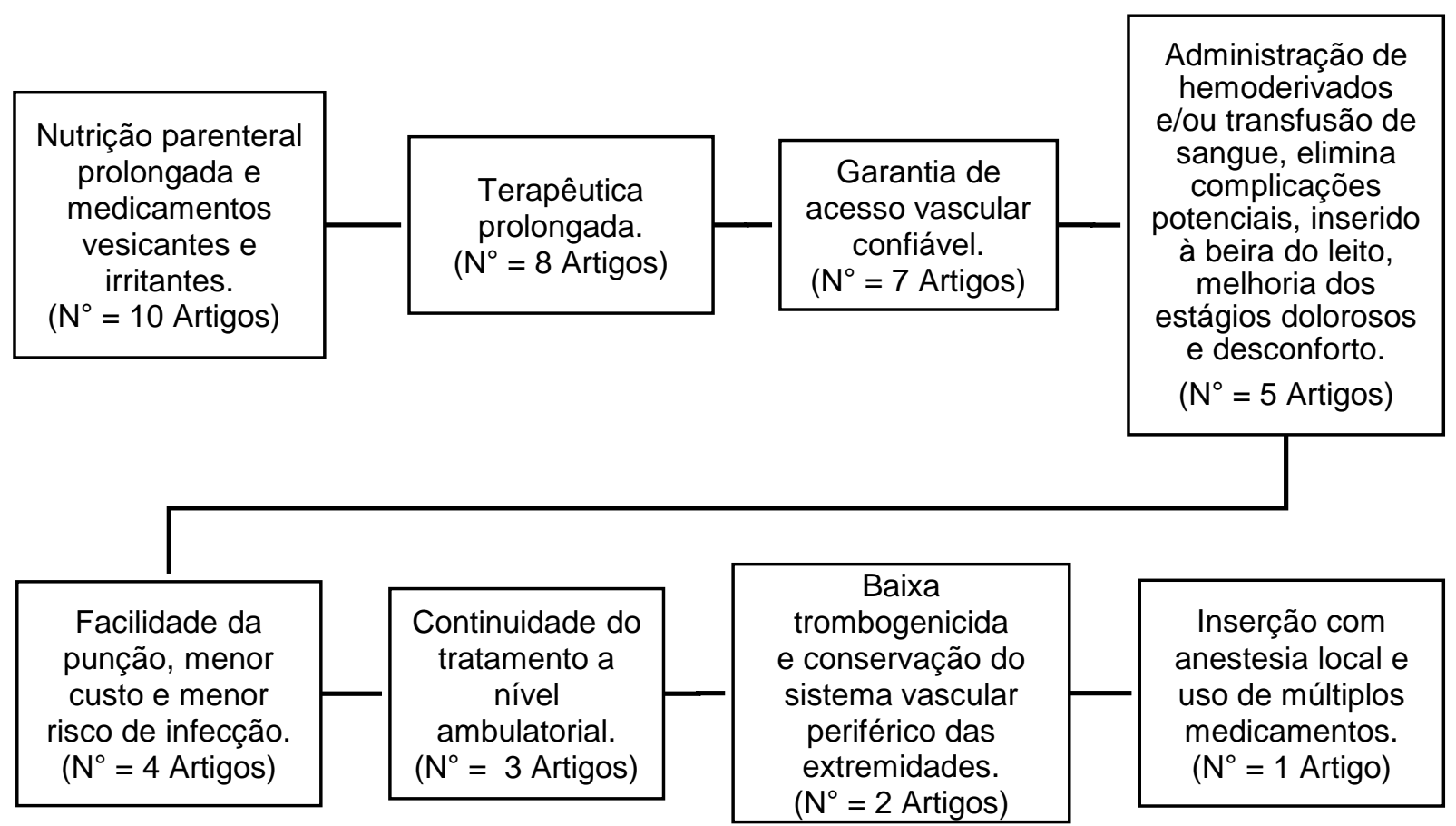

Fonte: Dados obtidos através da análise dos artigos selecionados.

Segundo Cotogni e Pittiruti (2014) para administração de dieta em PICC no multi lúmen é recomendado à utilização de um lúmen específico para sua oferta, e nunca se deve infundir fármaco ou qualquer infusão de $\mathrm{PH}$ divergente, pois se tem grandes possibilidades de complicações infecciosas, além disso, o recipiente de nutrição parenteral contendo dieta lipídica deve ser ofertada através do maior lúmen do dispositivo tendo em vista a possibilidade de obstrução do mesmo.

Entretanto em outro estudo considera critério benéfico para a implementação do PICC, nos pacientes em uso de nutrição parenteral total com concentração de soluções de glicose maior que $10 \%$, seguido pelo tempo de permanência prolongado do cateter, sendo adotado até o final da terapia intravenosa com um único PICC (VERA et al., 2015). Enquanto Jantsch et al. (2014) consideram adequado o uso do Cateter central de inserção periférica em concentrações de soluções de glicose maior que $12,5 \%$.

A primeira via de escolha de acesso vascular deve ser o PICC, pois em relação a outros dispositivos, ele fornece uma melhor assistência nas diversas terapêuticas empregadas até o término da terapia, além de possibilitar infundir soluções e drogas de $\mathrm{pH}$ ácidos ou alcalinos na veia cava superior e inferior, fazendo-lhe mais fluidas (LOPES et al., 2015).

Desde os anos 50 no século passado, foi observado que as drogas administradas através do PICC possuem maior dissolução reduzindo as chances de inflamação e trombose química (VERA et al., 2015). 
O cateter central de inserção periférica é indicado para tratamento prolongado em que o cliente necessita da administração de drogas irritantes e vesicantes por mais de uma semana, este dispositivo minimiza o risco de lesões cutâneas pelo extravasamento do fármaco e pode ser utilizado durante todo período terapêutico (SANTOS et al., 2017). O uso do PICC em pacientes oncológicos graves que necessitam de terapia intravenosa acima de seis dias, pode se prolongar de 30 dias até um ano em ambiente hospitalar, apesar da Infusion Nurses Society salientar que a retirada do cateter seja o mais precoce possível (NEGELISKII et al., 2017).

Já segundo Negeliskii et al. (2017) as complicações cutâneas são eventos que mais se repetem, entre elas destacam-se sinais flogísticos, infiltrações e extravasamentos, tal fatores contribuem para a diminuição da permanência do dispositivo.

Os autores Santo et al. (2017) descreve que a primeira escolha do acesso do PICC costuma ser na veia basílica direita. A veia cefálica possui a maior incidência de trombose venosa superficial sintomática com $7,2 \%$ em relação as demais veias, enquanto a veia braquial sua incidência é de $0 \%$, pois as incidências de trombose foram associadas aos vasos de diâmetro menor.

O PICC promove diminuição dos riscos de infecção de corrente sanguínea, é um dispositivo seguro, instalado através de uma punção de um vaso periférico em via central de grande calibre, pode ser passado por um enfermeiro devidamente habilitado, reduz gastos de recursos humanos e materiais, possui o valor de $\mathrm{R} \$$ 686,12 considerado baixo quando comparado ao cateter central e leva em média 40 minutos para sua colocação (NEGELISKII et al., 2017). Além disso, pode ser inserido a beira leito necessitando apenas de um raio$X$ para confirmação do local inserido (MOURA et al., 2013).

Dentre tais prerrogativas sobre o PICC a garantia de acesso vascular confiável, redução do desconforto e melhora dos estágios dolorosos são observados em pacientes oncológicos pediátricos que realizam tratamento prolongado em razão da diminuição de múltiplas punções venosas, favorecendo bem-estar e adesão efetiva ao tratamento (MOURA et al., 2013).

É de extrema importância que os pacientes oncológicos em terapia antineoplásico com quimioterapia ou nutrição parenteral domiciliar (HPN), assim como em cuidados paliativos não hospitalizados estejam em uso PICC como forma de facilitar a assistência a nível ambulatorial (COTOGNI e PITTIRUTI, 2014).

\section{CONCLUSÃO}

O uso do cateter central de inserção periférica (PICC) promove aos pacientes oncológicos pediátricos uma melhor qualidade de vida. O dispositivo tem como principal função reduzir os números de punções, tendo em vista à necessidade dos pacientes a submissão de tratamento quimioterápico prolongado. Possui menores riscos de complicações, menor custo em relação ao cateterismo venoso central de grandes vasos, é inserido a beira leito não necessitando do bloco cirúrgico para sua passagem, reduz a exposição desnecessária do paciente a infecções oportunistas, tendo em vista seu quadro imunológico que é considerado relativamente comprometido. Em alguns casos o cliente recebe alta em uso do cateter e é juntamente com seu familiar instruído a não manusear em ambiente domiciliar, tendo a necessidade de a cada 7 dias retornar ao ambulatório da oncologia para realização da troca de curativo. Diante dessas prerrogativas pudemos concluir que se faz necessário o treinamento e capacitação dos profissionais devidamente habilitados para passagem do dispositivo tendo em vista a diminuição das incidências no quadro de Infecções Relacionadas à Assistência à Saúde (IRAS).

\section{REFERÊNCIAS}

1. BRASIL. Agência Nacional de Vigilância Sanitária. Critérios diagnósticos de infecções relacionadas à assistência à saúde. $1^{a}$ ed. Brasília: ANVISA; 2013.

2. BRASIL. Instituto Nacional De Câncer José Alencar Gomes Da Silva. Câncer infantojuvenil. 2017.

3. BRASIL. Instituto Nacional De Câncer José Alencar Gomes Da Silva. Tipos de câncer infantil. 2018. 
4. BRASIL. Lei $\mathrm{n}$-9.610, de 19 de fevereiro de 1998. Altera, atualiza e consolida a legislação sobre direitos autorais e dá outras providências. Diário Oficial da República Federativa do Brasil, Brasília, DF, 20 de fev. 1998.

5. BRASIL. Ministério da Saúde. Conselho Nacional de Saúde. Resolução ํㅜ 510, de 7 de abril de 2016. Diário Oficial da República Federativa do Brasil, Brasília, DF, 24 maio 2016.

6. COTOGNI, P. et al. Peripherally inserted central catheters in non-hospitalized cancer patients: 5-year results of a prospective study. 2015; 23:403-409.

7. COTOGNI, P; PITTIRUTI, M. Focus on peripherally inserted central catheters in critically ill patients. World $\mathbf{j}$ crit care med. 2014; 3(4): 80-94.

8. GONÇALVES, KG. et al. Criança hospitalizada e equipe de enfermagem: opinião de acompanhantes. Rev enferm UFPE online. 2017; 11 SUPPL 6: 2586-2593.

9. JANTSCH, LB. et al. Utilização do cateter central de inserção periférica em neonatologia. Rev baiana enferm. 2014; 28(3): 244-251.

10. KARAM, PF. Cateter central de inserção periférica em oncologia pediátrica: desafios do cuidado no ambiente extra-hospitalar. Florianópolis: Universidade Federal De Santa Catarina. 2015. 127p.

11. LOPES, MLNC. et al. Utilização do cateter central de inserção periférica na unidade de terapia intensiva neonatal. Rev iberoam educ invest enferm. 2018; 8(1): 15-25.

12. MOURA, PF; CONTIM, D; AMARAL, JB. Cateter central de inserção periférica: revisão bibliométrica. Rev enferm atenção saúde. $2013 ; 2(1)$ : 106-115.

13. NEGELISKII, C. et al. Custo benefício do cateter central de inserção periférica em comparação com o cateter venoso central. Revista Estácio saúde. 2017; 6(1): 2-14.

14. OLIVEIRA, CR. et al. Cateter central de inserção periférica em pediatria e neonatologia. Esc Anna Nery rev enferm. 2014; 18(3): 379-385.

15. RANGEL, RL; FANDIÑO, M.C. Catéter central de acceso periférico en paciente pediátrico con cáncer: estudio de caso. Rev cuid. 2015; 6(1): 982-90.

16. RIBEIRO, JP. et al. Assistência de enfermagem ao paciente oncológico hospitalizado: diagnósticos e intervenções relacionadas às necessidades psicossociais e psicoespirituais. Rev pesqui cuid fundam online. 2016; 8(4): 5136-5142.

17. SANTOS, LMS. et al. Cuidados paliativos para a criança com câncer: reflexões sobre o processo saúde-doença. Rev bras pesqui saúde. 2013; 15(3): 130-138.

18. SANTO, MKD. et al. Cateteres venosos centrais de inserção periférica: alternativa ou primeira escolha em acesso vascular?. J vasc bras. 2017; 16(2): 104-112.

19. SANTOS, NF. et al. Competência e habilidade dos enfermeiros para a realização do PICC em crianças. Congresso internacional de enfermagem. $2017 ; 1(1): 1-3$.

20. SUNDRIYAL, D. et al. Peripherally inserted central catheters: our experience from a cancer research centre. Indian J Surg Oncol. 2014; 5(4):274-277.

21. UNESP. Biblioteca prof. Paulo de Carvalho Mattos. Tipos de revisão de literatura. 2015. Botucatu: Faculdade De Ciências Agronômicas.

22. VERA, SO; SOUSA, GN; ARAÚJO, SNM. A atuação do enfermeiro na prática de inserção e manutenção do picc: uma revisão integrativa de literatura. ReOnFacema. 2015; 1(1): 47-53. 